\title{
Comparative study on In-hospital Improvement of left Ventricular Ejection Fraction after PCI
}

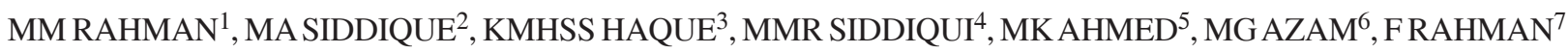

\begin{abstract}
:
The purpose of the study was to identify the impact of percutaneous coronary interventions (PCI) on ejection fraction between patients prior MI and without prior MI. This study was carried out at the University Cardiac Centre, Bangabandhu Sheikh Mujib Medical University (BSMMU), Dhaka, during the period between January 2006 to July 2006. Patients without history of prior MI experienced significant improvement of echo EF following PCI (62.30 \pm 5.52 vs. $46.63 \pm 6.43 \%, p<$ 0.001). In short term follow up remarkable improvement of LVEF is observed in patients of PCI who do not have prior MI in compared to patients having prior MI.
\end{abstract}

(University Heart Journal 2007; 3 : 60-62)

\section{Introduction:}

Cardiovascular disease is the most frequent cause of adult death worldwide. In many western countries the incidence of ischaemic heart disease (IHD) has been falling for the last two or three decades, but it is rising in eastern Europe and in Indian subcontinent, and this has led to predictions that cardiovascular disease will soon become the leading cause of death in all continents ${ }^{1}$. About 7.1 million deaths occurred globally in 1999 due to Coronary Heart Diseases and it will rise to 11.1 million by 2020 . WHO predicted that coronary heart disease (CHD) will be the top of the contributors to disease burden by 2020 and world will have to face the challenge of the crippling complications of the disease.

Now a day's percutaneous coronary intervention (PCI) is a commonly done procedure in the arena of interventional cardiology ${ }^{2}$. This has revolutionized the management of coronary artery disease. At present, the number of PCIs performed exceeds the number of coronary artery bypass graft (CABG) surgery almost everywhere ${ }^{3,4}$.

There are several options for treatment of CHD like nonpharmacologic, pharmacolgic, PTCA and surgical

1. Dr. Md. Mukhlesur Rahman, Asst. Prof, Dept. of Cardiology, UCC, BSMMU, Dhaka.

2. Prof. Md. Abu Siddique, Professor, Dept. of Cardiology, UCC, BSMMU. Dhaka.

3. Prof. KMHS Sirajul Haque, Professor \& Chairman, Dept. of Cardiology, UCC, BSMMU. Dhaka.

4. Dr. Md. Mahmudur Rahman Siddiqui, Postgraduate fellow, Dept. of Cardiology, UCC, BSMMU. Dhaka.

5. Dr. Md. Khurshed Ahmed, Asst. Prof. Dept. of Cardiology, UCC, BSMMU. Dhaka.

6. Dr. Md. Golam Azam, Consultant Cardiology, NICVD

7. Dr. Fazlur Rahman, Associate Professor, UCC, BSMMU. Dhaka. Address of correspondence: Dr. Md. Mukhlesur Rahman, Asst. Prof, Dept. of Cardiology, UCC, BSMMU, Dhaka. procedures. With the combination of sophisticated equipment, experienced operators, and modern drug therapy, percutaneous coronary interventions has evolved into an effective non-surgical modality of treatment ${ }^{5}$. Elizabeth (2005), demonstrated that patients with reduced Ejection Fraction (EF) have higher in-hospital mortality and other adverse outcome ${ }^{6}$.

\section{Materials and Methods:}

This study was carried out at the University Cardiac Centre, Bangabandhu Sheikh Mujib Medical University (BSMMU), Dhaka, during the period between January 2006 to July 2006. A total consecutive 60 patients were divided into two groups. Group-I $(n=32)$ included those patients having impaired left ventricular ejection fraction $(\mathrm{LVEF})<50 \%$, rest of the patients were included in group-II having preserved left ventricular ejection fraction (LVEF), ${ }^{350 \%}$. Patients with low EF undergoing PCI were the study population.

\section{Inclusion criteria:}

- Patients having prior MI with low ejection fractions undergoing PCI were taken as cases.

- Patients having low ejection fraction without prior MI were taken as control.

Exclusion criteria:

- Chronic renal failure (S. Creatinine level $>2.5 \mathrm{mg} / \mathrm{dl}$ ).

- Hepatic failure.

- Patients having valvular heart disease.

Methology:

- Informed written consent was taking from each patient.

- Detailed history was taken and thorough clinical examination were performed.

- Risk factor profile included-HTN, DM, dyslipidaemia, smoking and family history of CAD.

- Clinical profile to be recorded included pulse, BP, Angina pectoris (Stable or unstable), MI, Cardiac arrhythmias, peripheral vascular disease, $\mathrm{H} / \mathrm{O}$ stroke and functional status (NYHA class-I to IV)

- Echocardiographic variables specially included left ventricular ejection fraction (EF).

- Ejection fraction is measured by 2D and M-mode echocardiography by teichholz and simpson's rule (single plane). 
- LVEF were confirmed by ventriculography.

- $\quad$ Patients categorized according to the base line LVEF level- Cases-LVEF $<50 \%$, Control- LVEF ${ }^{350 \%}$

- Preprocedural angiographic variables including number of stenosed vessel (single / double /triple), degree of stenosis (0-100\%).

- Echocardiography was performed in all patients before discharge to see the improvement of LVEF after PCI.

\section{Results:}

A total of 60 patients of coronary artery disease were studied to assess the impact of ejection fraction on the outcome of PCI and the impact of PCI on EF. The results obtained from data analysis are presented below.

Table I

Age distribution of the patients $(N=60)$

\begin{tabular}{lll}
\hline Age $(\text { yrs })^{\#}$ & No & $\%$ \\
\hline$<40$ & 07 & 11.7 \\
$40-49$ & 18 & 30.0 \\
$50-59$ & 18 & 30.0 \\
$60-70$ & 13 & 21.7 \\
$\geq 70$ & 04 & 6.7 \\
\hline
\end{tabular}

\# Median age $=(50.0 \pm 10.5)$ years; range $=(32-75)$ year.

Table I shows that out of total 60 patients 18(30\%) were between the age of $40-49$ years and another $18(30 \%)$ were between 50 - 59 years of age. About $22 \%$ were between $60-70$ years, $11.7 \%$ below 40 years and only $6.7 \% 50$ years of age or above. The median age was found to be $50 \pm 10.5$ years and the lowest and highest ages being 32 and 75 years respectively.

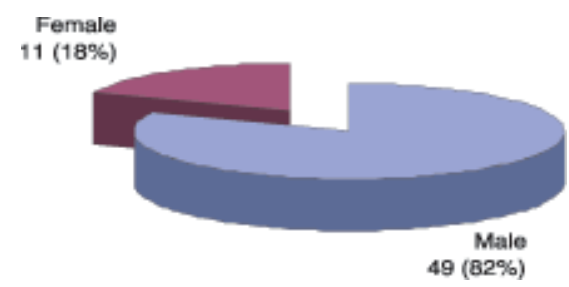

Fig. 1: Distribution of patients by sex

Fig. 1 shows that out of total 60 patients $49(82 \%)$ were male and the rest $11(18 \%)$ were female giving a male-female ratio of roughly $4.5: 1$.

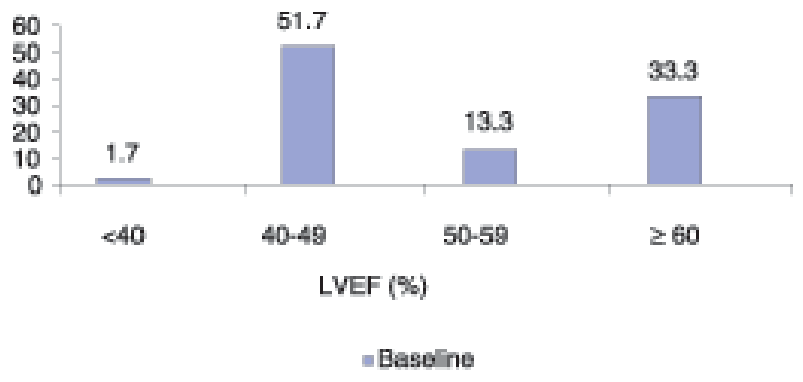

Fig. 4: Distribution of patients according to LVEF subgroups

Fig. 2 shows the distribution of patients by LVEF. More than half $(51.7 \%)$ of the patients had LVEF between 40 $49 \%$ followed by $33.3 \%$ had 60 or above $60 \%, 13.3 \%$ between $50-59 \%$ and only $1(1.7 \%)$ patient had below $40 \%$.

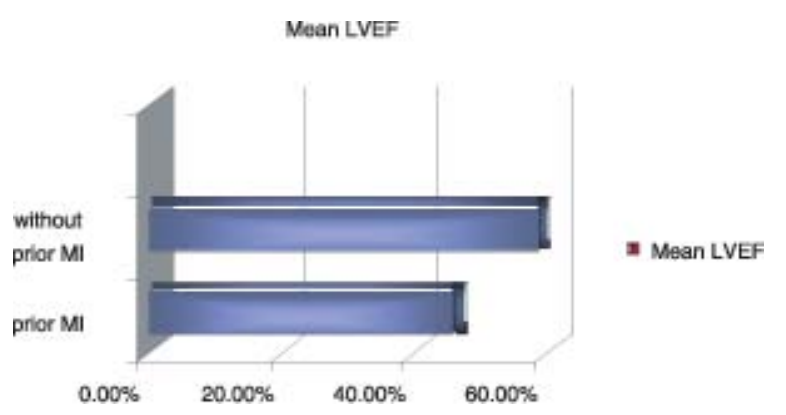

Fig: 3 shows mean LVEF before PCI in patient with prior MI was $45.08 \%$ and patient without prior MI was $54.75 \%$.

Table II

Comparison of post PCI LVEF between prior MI and without prior MI patients.

\begin{tabular}{lccc}
\hline Post PCI & Prior MI & p-value & \\
LVEF $(\%)^{\text {II }}$ & Yes $(\mathrm{n}=35)$ & $\mathrm{No}(\mathrm{n}=23)$ & \\
\hline LVEF following PCI & $46.63 \pm 6.43$ & $62.30 \pm 5.52$ & $<0.001$ \\
Improvement of LVEF & $0.08 \pm 0.21$ & $8.3 \pm 0.75$ & 0.637 \\
\hline
\end{tabular}

* Figures in the parentheses denote corresponding \%; S = Significant.

II Student's t-Test was employed to analyse the data and the level of significance was 0.05 .

Table XI: shows that subjects without history of prior MI experienced significant improvement of echo EF following PCI $(62.30 \pm 5.52$ vs. $46.63 \pm 6.43 \%, \mathrm{p}<0.001)$. 


\section{Discussion:}

This prospective study was carried out at the University Cardiac Centre, Bangladesh Sheikh Mujib Medical University (BSMMU), Dhaka, during the period between January 2006 to July 2006. A total consecutive 60 patients, who underwent to PCI with or without prior MI, included in this study and exclusion criteria were chronic renal failure, hepatic failure and valvular heart disease. patients were divided into two groups. Group-I $(n=32)$ included those patients having impaired left ventricular ejection fraction (LVEF) $<50 \%$ rest of the patients were included in group-II having preserved left ventricular ejection fraction (LVEF), ${ }^{350 \%}$. Data were collected in structured questionnaire, which contained all the variable of interest. The base line demographic, risk factor and haemodynamic status were recorded before and after procedure. Improvement of LVEF after PCI was measured in short term follow up period.

The mean age of the patients was $51.88 \pm 10.55$ yrs. The majority $(81.7 \%)$ of them were male. Patients without history of prior MI experienced significant improvement of echo EF following PCI (62.30 \pm 5.52 vs. $46.63 \pm 6.43 \%$, p $<0.001)$. In short term follow up remarkable improvement of LVEF is observed in patients of PCI who do not have prior MI in compared to patients having prior MI.

So, it may be concluded that, more attention should be paid to the ventricular ejection fraction of the patients before carrying out any coronary intervention. In patients having CAD, revascularization in the form of PCI should be performed as early as possible in patients having prior MI, revascularization should be performed after doing tests to detect viable myocardium. Randomized control trial should be conducted in future to determine the role of ejection fraction before PCI.

\section{Conclusion:}

Ischaemic heart disease (IHD) is an alarming health problem. Patients with IHD gradually develop congestive heart failure and ischaemic cardiomyopathy due to either ignorance or negligence about treatment ${ }^{7,8}$. Early revascularization not only prevent clinical deterioration but also improve $\operatorname{LVEF}^{9,10}$. In late stage, revascularization has little clinical benefit even associated with more procedural complications. In patients having CAD, revascularization in the form of PCI should be performed as early as possible in patients having prior $\mathrm{MI}$, revascularization should be performed after doing tests to detect viable myocardium. Randomized control trial should be conducted in future to determine the role of ejection fraction calculation in success of PCI .

\section{References:}

1. Mathew V, Grill D, Scott C, 'Baseline clinical and orgiographic variables associated with long term outcome after successful intracoronary stent implantatio' American Journal of Cardiology, 1999; 84: pp. 789-794.

2. Anderson RD, Ohman EM, Holmes Jr, 'Prognostic value of congestive heart failure history in patients undergoing Percutaneous coronary interventions' Journal of The American College of Cardiology, 1998 vol. 32, 936-941.

3. Ali MR, Haque KMSH S, Ahmed QS. 'Short-term outcome of precutaneous transluminal coronary angioplasty (PTCA) with and without stent in the treatment of coronary artery disease' Chest \& Heart Journal, 2002: 26(1), pp. 14-20.

4. Block PC, Peterson ED, Krone R, 'Identification of variables needed to risk adjust outcome of coronary interventions evidence based guideline for efficient data collection' Journal of The American College of Cardiology, 1988 vol. 32, pp. 275-282.

5. Holmes DR, Detre KM, 'Eight year, long term outcome after PTCA: factors associated with adverse events' The Nhlbi Registry, Journal of American College of Cardiology, 1996 vol. 27 , p. 361 .

6. Elizabeth R. 'The impact of ejection fraction on outcomes after percutaneous coronary intervention in patients with congestive heart failure: An analysis of the National Heart, Lung, and Blood Institute Percutaneous Transluminal Coronary Angioplasty Registry and Dynamic Registry' American Heart Journal, 2005 vol. 3, p. 53.

7. Haque S. 'Prevention and control of cardiovascular disease; Bangladesh perspective seminar on prevention and control of cardiovascular disease in Bangladesh' Souviner, JICA Alumni Association of Bangladesh, 2002 vol. 17, pp. 23-24.

8. Khalequzzaman M. 'Prevention of $C K-M B$ rise in percutaneous coronary intervention by prior treatment with beta blocker' MD thesis, National Institute of Cardiovascular Disease, 2003 Dhaka.

9. Smith SC, Dove JT, Jacobs AK. 'ACC/AHA guidelines for percutaneous coronary intervention (Revision of the 1999 PTCA guidelines), A report of the American College of Cardiology/ American Heart Association task force on practice guidelines (Committee to revised the 1999 guidelines for percutaneous transluminal coronary angioplasty)' Journal of The American College of Cardiology, 2001 vol. 37, p. 2239.

10. Malik A. 'Prevention of cardiovascular disease, Bangladesh Medical Journal, 1979; 7, pp. 91-93. 